J. Phys. IV France 130 (2005) 179-191

(C) EDP Sciences, Les Ulis

DOI: $10.1051 /$ jp4:2005130012

\title{
Dynamique moléculaire et canaux ioniques
}

\author{
S. Crouzy ${ }^{1}$
}

${ }^{1}$ Laboratoire de Biophysique Moléculaire et Cellulaire Département de Réponse et Dynamique Cellulaires, Centre d'Études Nucléaires de Grenoble, 17 rue des Martyrs, 38054 Grenoble Cedex 9, France

\begin{abstract}
Résumé. Diffusion de neutrons et Dynamique Moléculaire (DM) sont deux techniques intimement liées car elles portent sur les mêmes échelles de temps: la première apporte des informations structurales ou dynamiques sur le système physique ou biologique, la seconde permet de décoder ces informations à travers un modèle facilitant l'interprétation des résultats. Au delà de l'intérêt que la technique de DM peut avoir en relation directe avec les neutrons, il est intéressant de comprendre comment les modèles sont construits et comment les techniques de simulation peuvent aller beaucoup plus loin que de simples modélisations. Nous décrirons brièvement, dans la suite de cet exposé, la technique de DM et les méthodes plus sophistiquées de calculs d'énergie libre et de potentiels de force moyenne à partir des simulations de DM. Puis nous verrons avec deux exemples tirés de nos travaux théoriques sur les canaux ioniques comment ces calculs peuvent nous donner accès à des vitesses de réaction ou des constantes d'affinité ou de liaison. La première étude porte sur un analogue de la gramicidine A qui forme un canal conducteur d'ions interrompus par le basculement d'un cycle dioxolane [1]. La seconde concerne le canal potassique KcsA dont nous avons étudié le blocage du coté extracellulaire par l'ion Tetra Ethyl Ammonium [2].
\end{abstract}

\section{Table des matières}

1 Introduction

2 Technique de Dynamique Moléculaire

2.1 Mécanique et dynamique moléculaires . . . . . . . . . . . . . . . . . . . . 180

2.2 Calculs d'énergie libre . . . . . . . . . . . . . . . . . . . . 181

3 Basculement d'un cycle dioxolane dans un dimère $\begin{array}{ll}\text { de gramicidine } \mathbf{A} & 182\end{array}$

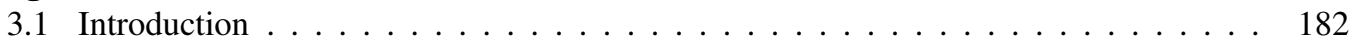

3.2 Modèle microscopique . . . . . . . . . . . . . . . . . . 183

3.3 Résultats . . . . . . . . . . . . . . . . . . . . . . . 184

4 Étude du blocage du canal potassique KesA par le TEA 186

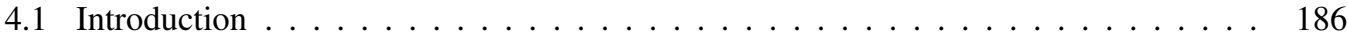

4.2 Modèles atomiques et méthodes de simulation $\ldots \ldots \ldots \ldots$. . . . . . . . 186

4.3 Résultats et discussion . . . . . . . . . . . . . . . . . . . 188

5 Conclusion $\quad 190$

\section{INTRODUCTION}

Les méthodes de Mécanique Moléculaire (MM) et Dynamique Moléculaire (DM) regroupent l'ensemble des outils permettant une étude structurale, dynamique ou énergétique de systèmes moléculaires. Toute modélisation de ces systèmes débute par la recherche des coordonnées spatiales des atomes constituant la macromolécule, dans des bibliothèques de structures obtenues par cristallographie aux rayons X ou Résonance Magnétique Nucléaire, telles que la Protein Data Bank. Aujourd'hui, MM et DM sont 
étroitement liées à l'application de techniques informatiques: Ainsi, les simulations et les visualisations sont réalisées sur ordinateur - in silico. La puissance des ordinateurs étant environ multipliée par deux tous les ans, on arrive maintenant à simuler des systèmes moléculaires complexes de plusieurs dizaines de milliers d'atomes sur des périodes de quelques dizaines de nanosecondes.

Parallèlement, depuis 1998, on a vu une progression remarquable du nombre de structures cristallographiques résolues de protéines membranaires telles que les canaux ioniques. Ces travaux ont été récompensés par l'attribution du prix Nobel de chimie en 2003 à l'équipe de Roderick MacKinnon, professeur de neurobiologie moléculaire et de biophysique à l'Université Rockefeller aux Etats-Unis pour la résolution de la première structure d'un canal ionique. Les canaux ioniques jouent un rôle essentiel, principalement dans le bon fonctionnement des cellules du système nerveux et des muscles squelettiques ou cardiaques. Structure et fonction des canaux ioniques sont demeurées longtemps méconnues, en raison de leur faible densité et du fait que ce sont des protéines membranaires intrinsèques se prêtant mal à la purification. L'équipe de MacKinnon réussit donc à déterminer la structure spatiale d'un canal sélectif aux ions potassiques, KcsA, en 1998. Peu après, cette même équipe obtint une structure de canaux ioniques sous deux états cinétiques distincts (ouvert ou fermé) puis la structure cristalline d'un canal ionique plus complexe, qui s'ouvre en réponse aux changements de potentiel. Il est maintenant possible de déterminer à l' échelle atomique les sites d'interaction des médicaments, et par conséquent de dessiner avec plus de précision les stratégies thérapeutiques applicables à de nombreuses maladies telles que les arythmies cardiaques, le diabète ou l'épilepsie. C'est dans cette nouvelle étape de recherche de sites d'interactions que la modélisation et la simulation de canaux ioniques prennent tout leur sens grâce à la possibilité qu' elles offrent de quantifier des différences d'affinité de liaison d'un médicament sur une cible donnée.

Après un bref rappel sur les bases de la DM, nous verrons, à travers deux exemples tirés de nos travaux sur les canaux ioniques, comment on peut simuler des profils d'énergie libre et prédire des taux de transition ou des affinités de liaison. La gramicidine A, tout d'abord, est un pentadécapeptide constitué d'acides aminés alternativement $\mathrm{L}$ et $\mathrm{D}$ qui se dimérise pour former un canal conducteur d'ions. Nous avons étudié [1] un analogue dans lequel les deux monomères sont liés de manière covalente par un cycle dioxolane en conformation RR ou SS, le RR pouvant seul basculer vers l'intérieur du canal et bloquer le passage des ions. Les simulations de DM ont permis de proposer un mécanisme de basculement du cycle et de calculer la vitesse de ce basculement en bon accord avec l'expérience. Le canal potassique KcsA, en second lieu, pour lequel l'équipe de Benoit Roux, principalement, a permis d'élucider les propriétes de conduction et de sélectivité [3,4] Nous mêmes nous sommes focalisés sur l'étude du blocage du canal KcsA par l'ion Tetra Ethyl Ammonium du coté extracellulaire [2]. Une mutation du canal en position 449 conduit à des comportements très différents en ce qui concerne le blocage par le TEA. Les simulations de DM permettent de calculer l'affinité du TEA pour les différents mutants du canal et d'expliquer le rôle particulier d'un résidu aromatique en position 449 .

\section{TECHNIQUE DE DYNAMIQUE MOLÉCULAIRE}

\subsection{Mécanique et dynamique moléculaires}

En mécanique moléculaire classique les atomes sont décrits par des masses ponctuelles $(m)$ et les liaisons chimiques par des ressorts. Les interactions entre particules sont décrites par une fonction d'énergie potentielle scalaire V. Toute particule est associée à un point de masse $(m)$ localisé, dont on connaît parfaitement, et à tout temps $t$, la position $\mathbf{r}(t)$ et le vecteur vitesse $\mathbf{v}(t)=\partial \mathbf{r}(t) / \partial t$. La présence du potentiel $V(\mathbf{r}, t)$ implique des forces (vectorielles), $\mathbf{F}(\mathbf{r}, t)$, qui sont directement opposées au gradient de $V$ au point $\mathbf{r}: \mathbf{F}(\mathbf{r}, t)=-\nabla_{\mathbf{r}} V(\mathbf{r}, t)$. Il convient donc de parler d'un champ de force à la place du potentiel. Une force ('la cause') agissant sur une particule d'une masse "inerte", $m$, va créer une accéleration, $\mathbf{a}$, de cette masse. L'équation de mouvement de Newton, base de la Dynamique Moléculaire, se formule 
comme suit:

$$
m \cdot \frac{\partial^{2} \mathbf{r}(t)}{\partial t^{2}}=-\nabla_{\mathbf{r}} V(\mathbf{r}, t)
$$

La position de la particule dans le temps, $\mathbf{r}(t)$, est appelé trajectoire.

La fonction d'énergie potentielle de CHARMM inclut 2 classes d'interactions moléculaires: i) Les interactions liées (définies entre deux ou plusieurs atomes connectés par des liaisons covalentes). ii) Les interactions non-liées (calculées entre tous les atomes qui ne sont pas directement connectés).

$$
V(\mathbf{r}, t)=E_{\text {total }}=\underbrace{E_{\text {liaison }}+E_{\text {angle }}+E_{\text {dièdre }}+E_{\text {impropre }}}_{\text {termes liés }}+\underbrace{E_{v d w}+E_{\text {elec }}}_{\text {termes non-liés }}
$$

Les termes liés $E_{\text {liaison }}, E_{\text {angle }}, E_{\text {impropre }}$ sont du type $K\left(x-x_{0}\right)^{2}$ et le terme $E_{\text {diedre }}$ est un terme en cosinus. L'énergie $E_{v d w}$ est approximée par un potentiel de Lennard-Jones comprenant un terme répulsif en $1 / r^{12}$ et un terme attractif en $1 / r^{6}$. Le terme d'énergie électrostatique, enfin, $E_{\text {elec }}$ est le potentiel classique de Coulomb en $1 / r$.

En pratique les équations de la DM sont résolues numériquement par la donnée de coordonnées et vitesses initiales pour tous les atomes et la résolution des équations de Newton 2.1 par un algorithme dont le plus utilisé est celui de Verlet. Après minimisation de l'énergie totale du système, une simulation de DM comprend trois phases: une période de chauffage pendant laquelle le système est porté lentement à sa température finale $T$ par modification des vitesses, une phase d'équilibrage pendant laquelle on laisse le système évoluer en n'intervenant que si la température dévie trop fortemment par rapport à $T$ et enfin la période de production où le système est totalement livré à lui même. C'est lors de cette dernière phase qu'est enregistrée la trajectoire qui pourra être analysée en détails lors de traitements ultérieurs.

Parmi ces analyses, on peut citer: calculs de structure moyenne et déviation par rapport à cette structure, calculs de corrélations entre mouvements atomiques, suivi des divers termes énergétiques du champ de force ou de mesures structurales (distances, angles...) en fonction du temps... Enfin, un des post-traitements les plus informatifs, mais aussi le plus long, est le calcul de différences d'énergie libre entre deux conformations visitées par le système ou le suivi du potentiel de force moyenne le long d'une coordonnée de réaction décrivant au mieux l'évolution du système.

\subsection{Calculs d'énergie libre}

L'énergie libre s'exprime théoriquement à partir de la fonction de partition notée $\mathrm{Z}$ qui décrit comment des particules se distribuent sur les divers états possibles. Dans le cas classique, on parle de fonction de partition configurationnelle, c'est à dire la fonction de partition évaluée en considérant les énergies potentielles $E(X)$ des différentes configurations $X$ d'un système de molécules

$$
Z=\int \ldots \int e^{-\beta E(X)} d X
$$

L'énergie libre $A$ vaut alors

$$
A=-k T \operatorname{Ln} Z
$$

Pour des systèmes relativement rigides, à peu de degrés de liberté, l'énergie libre peut être calculée directement à partir de Z. Cependant, pour des systèmes plus flexibles tels que des protéines dans l'eau, le mouvement de diffusion doit être pris en compte et $Z$ devient incalculable. De plus les énergies sont calculées par recherche de l'espace configurationnel; or, calculer l'entropie d'un système est complexe car seule la partie de l'espace configurationnel de basse énergie nous est accessible. Dans ces conditions, on peut obtenir de bonnes estimations de l'énergie interne et de l'enthalpie, pour lesquelles les basses énergies sont dominantes mais le calcul de l'entropie nécessite l'échantillonnage de régions de haute énergie. Cependant, nous sommes la plupart du temps intéressés par des différences d'énergie libre entre 
2 états notés 0 et 1 du type:

$$
\Delta A=A_{1}-A_{0}=-k T \operatorname{Ln} \frac{Z_{1}}{Z_{0}}
$$

Cette méthode de calcul, dite des "perturbations", est couramment utilisée dans les calculs de différences d'énergie libre entre deux états voisins de différence d'énergie $\Delta E$ (dont l'un peut être considéré comme une perturbation de l'autre) car on peut montrer que

$$
\Delta A=-k T L n\left\langle e^{-\beta \Delta E(X)}\right\rangle_{0}
$$

où le "0" signifie moyenne sur l'ensemble des configurations représentatives de l'état initial du système.

Afin de décrire un changement conformationnel en biologie ou une réaction chimique, il est nécessaire de choisir une ou plusieurs coordonnées de réaction $\xi$, capable de représenter la transformation de "réactants" vers "produits". Selon le choix de $\xi$, les barrières d'énergie à franchir peuvent varier du tout au tout. De plus, bien que la différence d'énergie libre entre "réactants" et "produits" ne dépende théoriquement pas du choix de $\xi$, les résultats pratiques obtenus à partir de calculs de dynamique moléculaire peuvent varier selon $\xi$.

Le potentiel de force moyenne $(\mathrm{PMF}) \mathcal{W}(\xi)$ le long de la coordonnée de réaction $\xi$ choisie, déduit de la différence d'énergie libre donnée par l'équation 2.3, s'exprime à partir de la fonction de distribution moyenne $\langle\rho(\xi)\rangle$,

$$
\mathcal{W}(\xi)=\mathcal{W}\left(\xi^{*}\right)-k_{B} T \ln \left[\frac{\langle\rho(\xi)\rangle}{\left\langle\rho\left(\xi^{*}\right)\right\rangle}\right]
$$

où $\xi^{*}$ et $\mathcal{W}\left(\xi^{*}\right)$ sont des constantes arbitraires.

La méthode des perturbations ne fonctionne, on l'aura compris, que si l'on est capable d'échantillonner correctement l'état initial et l'état final de la réaction. Si ces états sont trop éloignés (en énergie), on découpe le chemin qui les relie en plusieurs fenêtres et on recombine, comme il se doit, les $\mathcal{W}(\xi)$ partiels.

Mais, quelquefois, ce découpage est insuffisant car la barrière d'énergie entre deux états est trop importante. On utilise alors la méthode de l'échantillonnage avec biais harmonique umbrella sampling de Torrie et Valleau [5]. Dans cette méthode, le système microscopique est simulé en présence d'un potentiel de biais harmonique artificiel $w(\xi)$, introduit pour améliorer l'échantillonnage au voisinage d'une valeur choisie $\xi$ de la coordonnée de réaction. Les équations WHAM expriment alors l'estimateur optimal de la fonction de distribution débiaisée comme une somme pondérée sur les $N_{\mathrm{w}}$ fonctions de distributions biaisées individuelles [6].

\section{BASCULEMENT D'UN CYCLE DIOXOLANE DANS UN DIMÈRE DE GRAMICIDINE A}

\subsection{Introduction}

Le canal ionique formé par la molécule de gramicidin A, représenté sur la Fig. 1 est un pentadécapeptide linéaire produit par bacillus brevis constitué d'acides aminés alternativement L et D HCO-Val-GlyAla-D-Leu-Ala-D-Val-Val-D-Val-Trp-D-Leu-Trp-D-Leu-Trp- NHCH2CH2OH qui est encore à l'heure actuelle l'objet de nombreuses études tant expérimentales que théoriques.

Le canal conducteur d'ions est un dimère N-Ter-N-Ter (tête à tête), formé par deux hélices droites $\beta$-6.3 mono-brin, stabilisé par la formation de 6 liaisons hydrogène inter-monomère $\mathrm{NH}-\mathrm{O}$.

Un processus bimoléculaire impliquant l'association et la dissociation des monomères est responsable de l'ouverture et de la fermeture du canal dimérique naturel. Un analogue du canal particulièrement intéressant dans lequel les 2 monomères ont été liés côté $\mathrm{N}$-terminal par un cycle dioxolane a été synthétisé par Stankovic et coll. [8]. Les 2 stéréoisomères de gramicidine liée par cycle dioxolane (GADiox) ainsi produits, les formes SS et RR, ont montré des propriétés très différentes. Des interruptions rapides et 

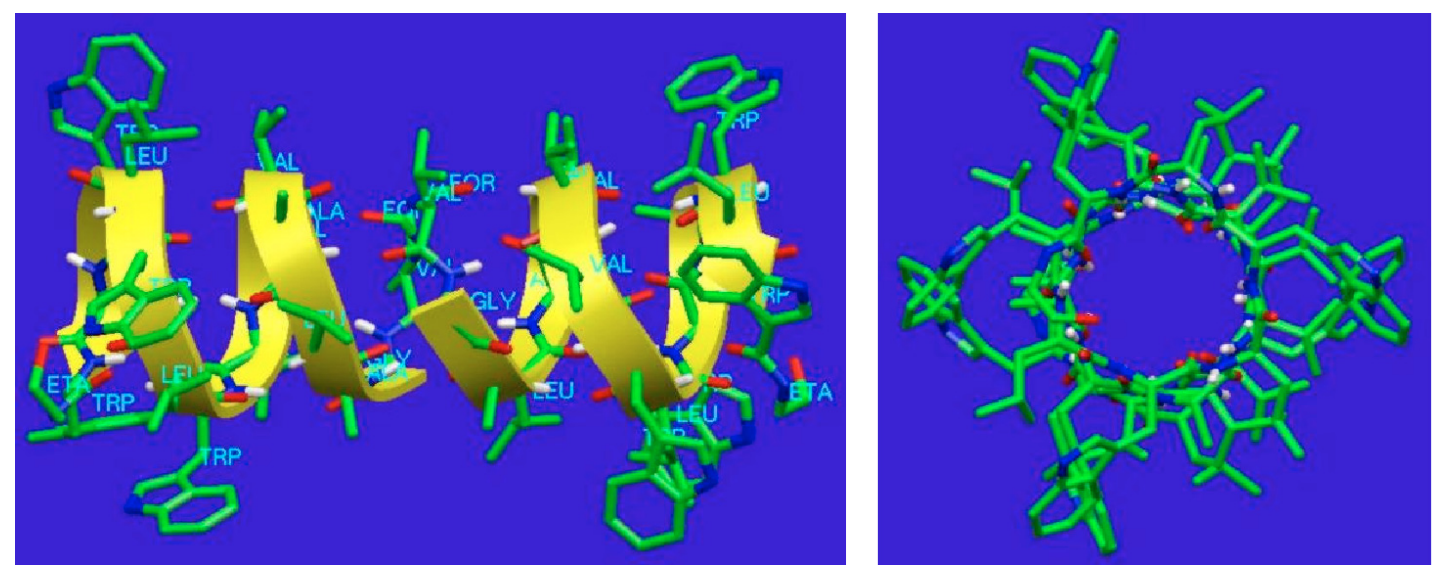

Figure 1. Structure de la gramicidine représentée avec le programme MPV3D [7]: A gauche, vue transverale, à droite, vue dans l'axe du pore.

fréquentes du courant ont été observées lors d'expériences de Patch-Clamp en canal unique sur la forme RR et non sur la forme SS. De plus l'échelle de temps des interruptions mesurées avec la forme RR est proche de celle rencontrée dans le cas de canaux biologiques; le taux de passage dans l'état fermé est de 100 fois par seconde et la durée moyenne de séjour dans l'état fermeé est de $0.1 \mathrm{~ms}$. De telles interruptions brèves, absentes des enregistements réalisés sur des canaux de gramicidine naturelle ont été interprétées comme une preuve de l'existence de transitions conformationnelles du cycle dioxolane de l'extérieur vers l'intérieur du canal, jouant ainsi le rôle de "porte" ouvrant ou fermant le canal. L'étude que nous avons menée [1] consistait donc à comprendre les mécanismes microscopiques du basculement du cycle dioxolane en supposant que la structure tri-dimensionnelle globale du dimère en hélice $\beta$ était conservée dans le canal GADiox.

\subsection{Modèle microscopique}

Les canaux GADiox RR et SS ont été construits à partir des coordonnées de la structure en hélice droite d'Arseniev et Coll. [9]. Un cycle 1,3-dioxolane (C1 C2 O3 C4 C5) est venu en substitution des groupes formyl côté $\mathrm{N}$-terminal de chaque monomère de gramicidine et des liaisons chimiques ont été créées avec le résidu Val1. Douze molécules d'eau ont été disposées en file le long de l'axe du canal, distantes de $2.8 \AA$, les 2 molécules d'eau centrales, 6 et 7 , entourant le cycle lorsqu'il est à l'intérieur. La protéine est représentée dans un modèle atome étendu dans lequel les hydrogènes non polaires sont absents ce qui donne un système total de 319 atomes. La fonction de potentiel empirique décrivant les interactions ion-canal comporte 2 modifications par rapport aux termes énergétiques standards. La répulsion à courte distance entre $\mathrm{K}^{+}$et les oxygènes des carbonyls est décrite par un potentiel en $1 / r^{8}$ moins raide que le potentiel de Lennard Jones en 6-12 et un terme de polarisation du premier ordre a été introduit pour les interactions ion-peptide. Les polarisabilités atomiques appliquées à tous les atomes sauf les hydrogènes ont été ajustées pour reproduire les résultats de calculs ab-initio: alpha $=0.3$ pour $\mathrm{C}, 0.5$ pour les groupes méthyls, 1.5 pour les $\mathrm{N}$ et les $\mathrm{O}$ à l'exception de ceux du cycle dioxolane pour lesquels alpha $=0.5$. Aucune polarisabilité n'a été rajoutée pour les molécules d'eau. Les autres paramètres non liés utilisés pour le cycle dioxolane ont été tirés du champ de force OPLS. Un faible potentiel de confinement semi-harmonique a été appliqué aux oxygènes des 3 molécules d'eau les plus excentrées de chaque côté du canal pour les confiner près de l'entrée. Les interactions non-liées ont été calculées atome par atome avec une coupure à $12 \AA$ réduite à $9 \AA$ pour les calculs de PMF pour gagner du temps de calcul. Les interactions électrostatiques utilisent un epsilon de 1 et sont adoucies par une fonction "shift" tandis que les interactions de van der Waals utilisent une fonction switch sur une distance de $1 \AA$. 
Le centre de masse du dimère est placé à l'origine et le canal orienté selon l'axe Z. Les trajectoires sont générées par dynamique de Langevin à $300 \mathrm{~K}$ avec une constante de friction correspondant à un temps de relaxation de $20 \mathrm{ps}^{-1}$ appliquée sur tous les atomes de carbone sauf ceux du cycle dioxolane pour assurer un échantillonnage conformationnel de Boltzmann caractéristique de l'ensemble canonique. Un pas de temps d'intégration de 1 fs a été utilisé.

\subsection{Résultats}

Suivant des études préliminaires, le basculement du cycle dioxolane avait été décrit par une coordonnée de réaction $q(R)$ égale au cosinus entre deux vecteurs $\mathbf{u}$ et $\mathbf{e}$. u est le vecteur reliant le centre de masse de la gramicidine et le point milieu des deux carbones $\alpha$ des valines 1 et e est le vecteur reliant le point milieu entre les deux atomes $\mathrm{C} 1$ et $\mathrm{C} 2$ du cycle et l'atome $\mathrm{C} 4$ du cycle. $Q$ semblait une coordonnée tout à fait adaptée à l'étude du basculement du cycle avec des valeurs variant entre -1 pour le cycle à l'intérieur et +1 pour le cycle à l'extérieur du canal. Cependant, le calcul de PMF sur la seule base de la coordonnée $q$ s'est avéré impossible. En particulier, on observait fréquemment une isomérisation du lien peptidique dioxolane-Val1 de sa valeur de départ trans vers cis lors de la génération des trajectoires nécessaires au calcul du PMF le long de $q$. Généralement, ces difficultés sont le signe que la coordonnée de réaction choisie n'est pas suffisante pour fournir une description complète de la transition dynamique dans le système. Il aparaissait donc que $q$ et l'angle dièdre de la liaison peptidique avec Val1, $\omega$, étaient tous 2 impliqués dans la transition du cycle.

De manière idéale, les corrélations entre $q$ et $\omega$ devraient être prises en compte en calculant un PMF bidimensionnel fonction des 2 variables. Comme ces calculs auraient été beaucoup trop coûteux, des surfaces d'énergie adiabatiques en fonction de $q$ et $\omega$ ont pu être calculées pour mettre à jour les barrières d'énergie potentielle dominantes contrôlant les transitions conformationnelles du système. Ces cartes adiabatiques sont visibles sur la Fig. 2.

RR
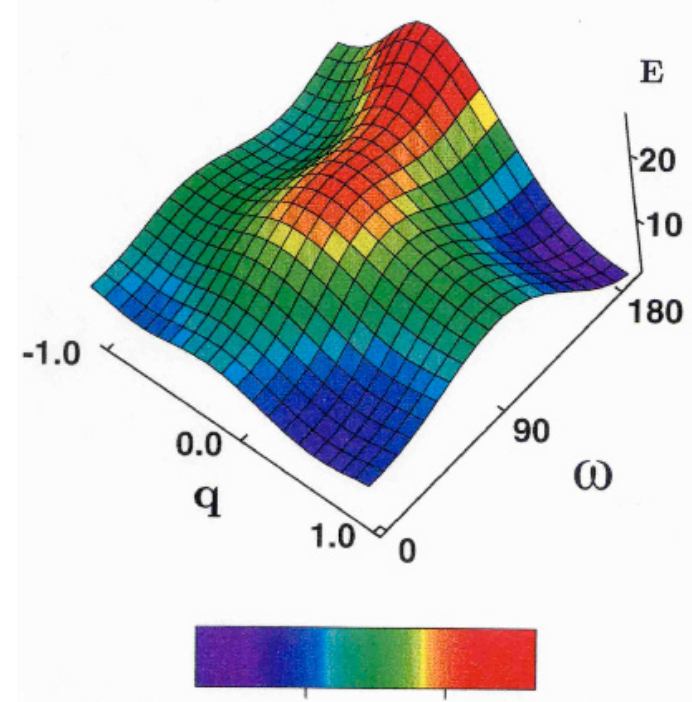

10
SS
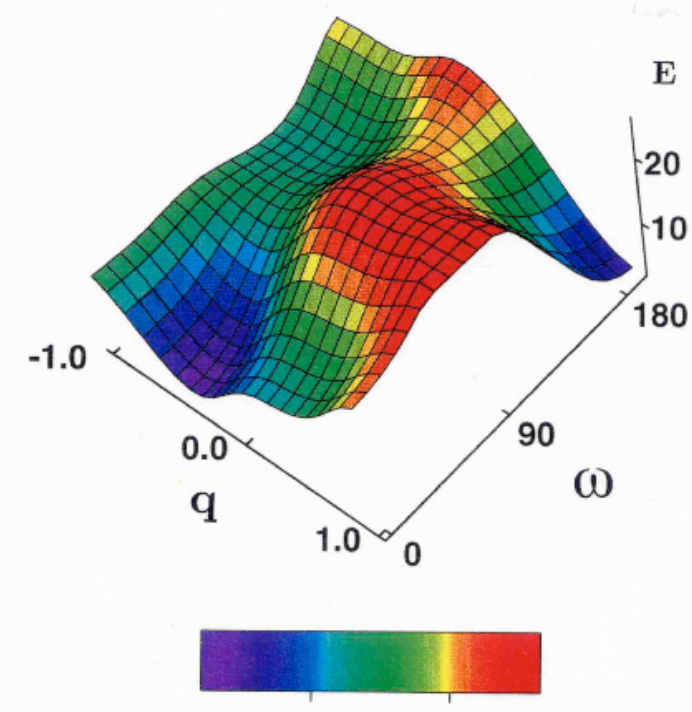

10

Figure 2. Profils d'énergie adiabatique tri-dimensionnels pour le cycle en conformation RR et SS. 
Comme les configurations peuvent rester piégées dans des minima locaux lors de l'optimisation de géométrie, les surfaces calculées peuvent présenter des discontinuités et des artefacts. Pour éliminer le plus possible le problème des minima locaux, ces cartes ont été calculées sans molécules d'eau et nous avons mis au point une technique particulière appelée AMRP pour Automatic Map Refinement Protocol [10]. L'observation des cartes montre que l'état out-trans correspond à un minimum de plus basse énergie aussi bien pour la conformation RR que la conformation SS du cycle dioxolane. Les états Out-cis, In-cis et In-trans correspondent eux aussi à des minima d'énergie locaux l'état In-trans présentant la plus haute énergie. Out trans est le minimum dans le cas RR tandis que In-trans est plus bas de $0.7 \mathrm{kcal} / \mathrm{mol}$ dans le cas SS. Des barrières d'énergie importantes séparent l'état Out-trans des états Out-cis et In-trans dans le cas SS. Au contraire, un chemin favorable Out-trans Out-cis In-cis In-trans semble se dessiner dans le cas RR qui suggère une voie permettant au cycle dioxolane de rapidement entrer à l'intérieur du canal et bloquer le passage des ions. Ainsi ces cartes montrent-elles que l'isomérisation de l'angle dièdre $\omega$ était bien nécessaire pour faciliter la pénétration du cycle vers l'intérieur en conformation RR.

Pour le cycle SS, les calculs de PMF font apparaitre une barrière d'énergie libre de $31 \mathrm{kcal} / \mathrm{mol}$ pour la transition Out-trans $\rightarrow$ Out-cis et de $21 \mathrm{kcal} / \mathrm{mol}$ pour la transition directe Out-trans $\rightarrow$ In-trans.

Les informations expérimentales concernant la dynamique de la transition ouverture/fermeture des canaux de gramicidine liée par le dioxolane ont été obtenues par la technique d'enregistrement de canal unique dans des membranes de GMO/squalene [8]. Pour l'isomère RR, de brèves fermetures durant en moyenne $0.1 \mathrm{~ms}$ ont été observées en moyenne 100 fois par seconde. Aucune interruption de courant n'a pu être observée pour l'isomère SS tandis que les simulations donnent une fréquence de fermeture de 10 par ms et une durée moyenne de fermeture de $0.06 \mu$ s pour la transition limitante de Out-trans vers Out-cis; ces 2 résultats ne sont pas incompatibles car des interruptions de courant aussi courtes n'auraient vraissemblablement pas pu être vues expérimentalement. Dans le cas de l'isomère RR, le chemin le plus favorable énergétiquement pour passer de l'état conducteur à l'état bloqué est Out-trans $\rightarrow$ Out-cis $\rightarrow$ In-cis $\rightarrow$ In-trans. L'étape la plus lente est la transition Out-trans $\rightarrow$ Out-cis qui correspond à l'isomérisation du lien peptidique de $\mathrm{Val}^{1}$ avec le cycle dioxolane à l'extérieur du canal. L'étape limitante pour le taux de réaction qui détermine la durée de vie de l'état fermé est la transition In-trans $\rightarrow$ In-cis correspondant à l'isomérisation du lien peptidique de $\mathrm{Val}^{1}{ }^{1}$ avec le dioxolane à l'intérieur et dont le profil d'énergie est montré sur la Fig. 3. Le taux de blocage et la durée moyenne de fermeture sont estimées à $280 /$ s et 9 ns respectivement. La durée moyenne de vie de l'état fermé est en désaccord complet avec les observations expérimentales. Quelle peut en être la cause? L'inexactitude de la fonction d'énergie dans le champ de force n'est sans doute pas le facteur dominant; par contre l'influence possible de l'environnement aqueux, l'absence de lipides et surtout l'absence d'ion perméant dans le canal sont probablement à la source du problème. Rappelons que les mesures expérimentales ont été faites en

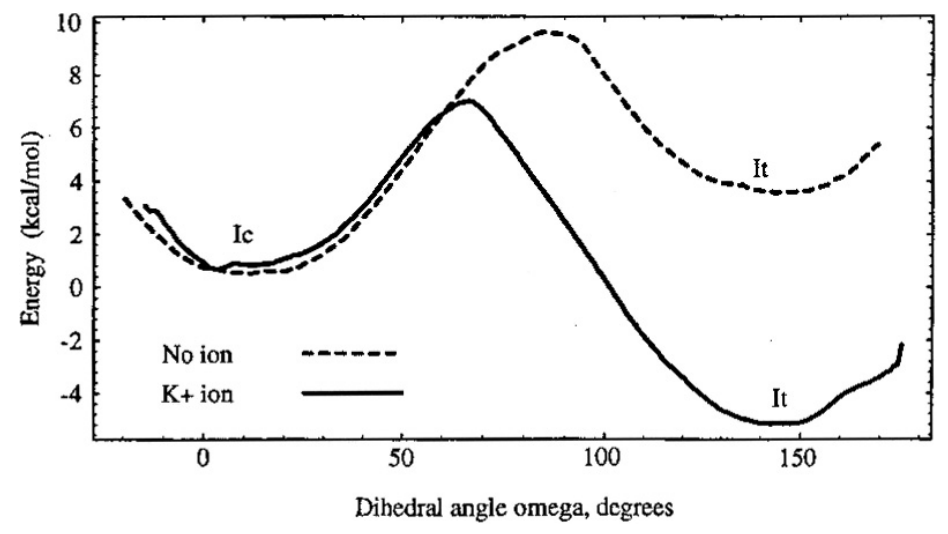

Figure 3. Profils d'énergie libre pour le cycle en conformation RR. 
présence d'une forte concentration en ion $\mathrm{K}^{+}(640 \mathrm{mM} \mathrm{KCl})$ et la probabilité qu'au moins un ion se trouve à l'intérieur du canal est estimée à $97 \%$ d'après une analyse par Heineman et Sigworth [11]. De nouveaux PMF ont donc été calculés après introduction d'un ion $\mathrm{K}^{+}$dans le pore. L'isomérisation du lien peptidique avec le dioxolane à l'intérieur du canal reste l'étape limitante qui détermine la durée de vie de l'état fermé et le profil d'énergie correspondant est montré sur la Fig. 3. On observe que l'ion $\mathrm{K}^{+}$ près du centre du canal stabilise considérablement l'état In-trans. La barrière d'énergie d'activation passe de $5.9 \mathrm{kcal} / \mathrm{mol}$ en absence d'ion à $12.2 \mathrm{kcal} / \mathrm{mol}$ et la durée moyenne de l'état fermé passe à $0.04 \mathrm{~ms}$. Le cycle RR passerait alors $97 \%$ du temps hors du canal et $3 \%$ à l'intérieur ce qui est en bon accord avec les données expérimentales.

\section{4. ÉTUDE DU BLOCAGE DU CANAL POTASSIQUE KCSA PAR LE TEA}

\subsection{Introduction}

Une part importante des connaissances actuelles sur la fonction des canaux potassium a été déduite d'études avec des bloqueurs du pore basés sur des ammonium quaternaires (QA), tels que le tetraméthylammonium (TMA), le tetraéthylammonium (TEA), le tetrabutylammonium (TBA), et autres dérivés alkyles [12]. Par leur capacité à bloquer fortement les canaux potassiques, les QA sont des outils importants dans les études électrophysiologiques des membranes biologiques. Les études abondent dans le sens d'une action de ces agents par blocage de la voie de perméation, empêchant le mouvement des ions $\mathrm{K}^{+}$à travers le pore. Presque tous les canaux $\mathrm{K}^{+}$sont sensibles à un blocage par les QA du côté interne de la cellule. Par contre, certains canaux $\mathrm{K}^{+}$sont sensibles au blocage par le TEA du côté externe, tandis que d'autres sont insensibles. La liaison du TEA est particuliérement affectée par la présence ou non d'un résidu aromatique en position 449 du type Shaker, supposé situé près de l'entrée du pore [13]. De plus, le site de liaison externe est très sélectif pour le TEA par rapport au TMA. La détermination récente de la structure tri-dimensionnelle du canal KcsA par cristallographie aux rayons X [14] a remis au goût du jour certaines questions concernant l'interprétation antérieure de données électrophysiologiques sur le blocage des canaux $\mathrm{K}^{+}$par le TEA appliqué côté externe [13]. Comme le Shaker, KcsA est sensible à un blocage par le TEA du côté externe [15]. De plus, à partir d'une combinaison de données structurales et fonctionnelles en présence de neurotoxines de scorpion, on a pu conclure que KcsA est structurellement très similaire aux canaux $\mathrm{K}^{+}$eucaryotes comme le Shaker [16]. Quelle est donc la nature des interactions microscopiques du TEA impliquées dans la spécificité du blocage externe? Des considérations basées sur une vue statique du canal sont vraissemblablement insuffisantes. En particulier, l'influence de l'environnement aqueux et de la dynamique du canal peuvent jouer un rôle important sur le blocage par le TEA comme nous allons le résumer dans la suite à partir de nos travaux récents [2].

\subsection{Modèles atomiques et méthodes de simulation}

Toutes les simulations de MD ont été menées avec le programme CHARMM [17] en utilisant la champ de force tous-atomes décrit par le fichier de paramètres PARAM22 [18] pour les protéines et les lipides [19] La molécule de TEA de charge +1 a été paramétrisée pour être compatible avec PARAM22. Le TEA peut adopter deux conformations principales: totalement symétrique (ou quasi-planaire) et assymétrique (pyramidale). Des calculs de mécanique quantique $A b$ initio indiquent que le conformère symétrique est plus stable de $0.9 \mathrm{kcal} / \mathrm{mol}$; La contribution électrostatique à l'énergie libre de solvatation du TEA a été calculée selon la méthode des perturbations, par simulations du TEA dans une sphère de 120 molécules d'eau explicites en présence du potentiel de frontière sphérique pour le solvant (SSBP) [20]. L'énergie calculée nécessaire pour charger le TEA est environ de $-49 \mathrm{kcal} / \mathrm{mol}$, en excellent accord avec la valeur expérimentale de $-49 \mathrm{kcal} / \mathrm{mol}$ [21] (la petite énergie libre positive de création de la cavité non-polaire a été négligée). La différence d'énergie libre de "charge" entre les conformères symétrique et assymétrique est d'environ $0.9 \mathrm{kcal} / \mathrm{mol}$, en faveur du conformère symétrique. La combinaison des 
résultats précédents $a b$ initio et énergie libre de solvatation donne un conformère symétrique plus stable par $1.8 \mathrm{kcal} / \mathrm{mol}$. Cette différence d'énergie se traduisant en $95 \%$ de conformères symétriques contre $5 \%$ d'assymétrique, c'est le conformère symétrique qui a été choisi dans les simulations. Des modèles atomiques de KcsA avec le TEA bloquant côté externe ont été construits pour un canal sauvage (KcsAY82) et un canal muté dans lequel Tyr82 est remplacée par une Thr (KcsA-T82). Le résidu substitué se trouve juste après la séquence signature commune à tous les canaux $\mathrm{K}^{+}$:TTVGYGDM-T(449) pour le Shaker, et TTVGYGDL-Y(82) pour le KcsA. La configuration initiale du système utilisée ici provient d'une configuration équilibrée du canal $\mathrm{K}^{+} \mathrm{KcsA}$ inséré dans une membrane de DPPC dans une solution saline contenant $150 \mathrm{mM} \mathrm{KCl} \mathrm{[3].} \mathrm{Le} \mathrm{protocole} \mathrm{général} \mathrm{de} \mathrm{construction} \mathrm{et} \mathrm{d'équilibration} \mathrm{de} \mathrm{ce} \mathrm{système}$ initial a été décrit en détail [3]. Le mutant Y82T a été construit à partir du système sauvage équilibré en faisant correspondre les coordonnées des atomes identiques (squelette et $\mathrm{C} \beta$ ) et en construisant les atomes restants des chaines latérales des Thr82 dans une géométrie coorespondant au rotamère le plus probable, à partir des coordonnées internes. Le nombre total d'atomes dans le système visible sur la Fig. 4 est proche de 40000.

Le système entier est électriquement neutre. L'axe du canal est orienté le long de l'axe $Z$; le centre de la membrane est en $Z=0$.

Le TEA a été placé côté extracellulaire des deux modèles de canaux (KcsA-Y82 et KcsA-T82), à environ 20.4 Å sur l'axe $Z$. Les molécules d'eau en contact avec le TEA ainsi que l'ion $\mathrm{K}^{+}$du site externe (situé à $\mathrm{Z}=15 \AA$ A) ont été retirés; l'ion $\mathrm{K}^{+}$de la cavité a été conservé. Après construction, les modèles atomiques ont été raffinés par minimisation d'énergie et équilibrés pendant 450 ps. Des trajectoires de

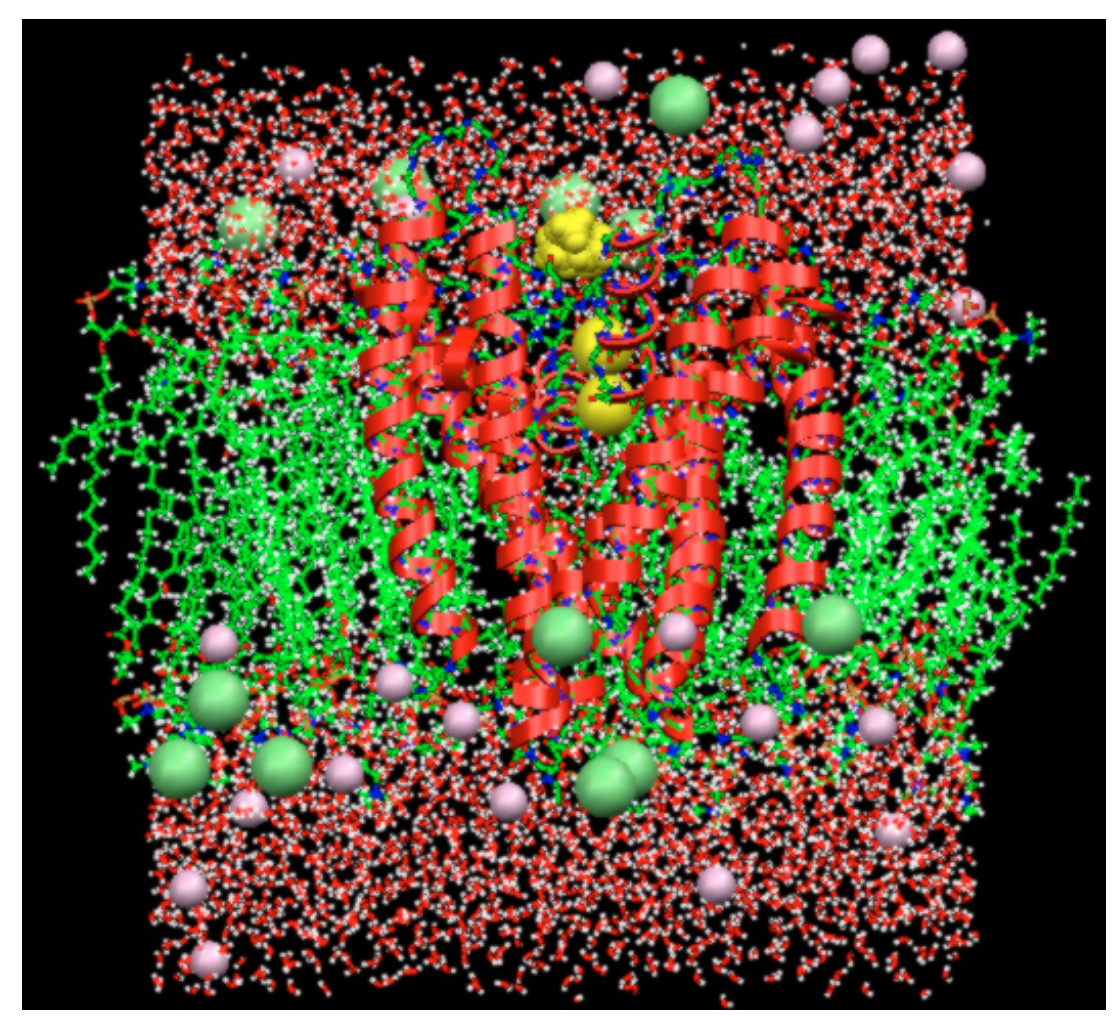

Figure 4. Vue du système total KcsA (hélices rouges), TEA (jaune), 112 DPPC, 6532 molécules d'eau, $2 \mathrm{~K}^{+}$dans le pore (jaune) et $12 \mathrm{~K}^{+}$et $23 \mathrm{Cl}^{-}$dans la solution. La partie $\mathrm{Y}>0$ des lipides et du solvant a été retirée pour plus de clarté. 
1.75 (Y82-MD1) et 1.2 ns (T82-MD1) ont été produites à $315 \mathrm{~K}$ pour les systèmes KcsA-Y82 et KcsAT82, respectivement La simulation du canal muté Y82T a été arrêtée après que le TEA fut sorti du canal dérivant dans le solvant. Les deux trajectoires ont été générées à pression constante avec conditions aux limites périodiques [22]. Les interactions électrostatiques ont été calculées sans coupure avec la méthode "particle mesh ewald" (PME) [23]. Le potentiel de force moyenne a été calculé par la méthode de "l'échantillonnage avec biais harmonique [6] décrite précédemment. Ici, les simulations ont été générées en présence d'un potentiel harmonique de la forme $K\left[r-r^{(i)}\right]^{2}$ : où $r$ est la distance entre le centre de masse du TEA et le centre de masse du squelette des résidus Thr75-Val76-Gly77-Tyr-78 qui constituent le coeur du filtre de sélectivité. 15 simulations indépendantes ont été générées avec des potentiels de biais harmonique centrés successivement en $r^{(i)}$ égal à $8.5,9.0, \ldots$, et $15.5 \AA$. avec une constante de force de $K=10 \mathrm{kcal} / \mathrm{mol} / \AA^{2}$. Le système initial a été construit avec deux ions $\mathrm{K}^{+}$dans le filtre de sélectivité d'après les résultats obtenus sur les simulations sans contrainte. Après construction, les modèles ont été raffinés par minimisation d'énergie et équilibrés pendant 200 ps avec le TEA côté extracellulaire comme précédamment. Le protocole de génération des fenêtres comprend une période d'équilibration de 10 ps suivie par la période de production et acquisition des données. Le PMF du type sauvage KcsA-Y82, $\mathcal{W}_{\text {Y82 }}(r)$, a été calculé à partir de simulations de MD de 114 ps par fenêtre auxquelles s'ajoutent 54 ps pour la région centrale $\left(9.5 \leq r^{(i)} \leq 12.5 \AA\right.$ ) pour une meilleure convergence (simulation totale de 168 ps). Le PMF pour le canal mutant $\operatorname{KcsA}-\mathrm{T} 82, \mathcal{W}_{\mathrm{T} 82}(r)$, quant à lui, a été calculé à partir de simulations de MD de 124 ps par fenêtre. La méthode WHAM a été utilisée pour débiaiser et recombiner les résultats des 15 simulations individuelles biaisées de manière optimale [6]. Enfin, en complément des résultats obtenus sur l'état lié, d'autres trajectoires de 0.5 ns ont été générées en partant d'un TEA dans les puits d'énergie déterminés sur les PMF pour les canaux KcsA-Y82 et KcsA-T82 sans potentiel de biais. Ces trajectoires seront appelées Y82-MD2 et T82-MD2. De plus, une simulation de référence de 100 ps du TEA dans une sphère de $20 \AA$ contenant 1064 molécules d'eau a été générée en présence du potentiel SSBP pour calculer la fonction de distribution radiale du solvant. Tous les calculs ont été réalisés sur une SGI Origin au NCSA, sur un cluster de PC Linux, et sur un supercalculateur COMPAQ SC232 au CEA à Grenoble. Une ns de simulation a pris environ 250 heures sur la SGI Origin avec 16 CPU R10000 calculant en parallèle avec l-interface MPI (Message Passing Interface).

\subsection{Résultats et discussion}

Les résultats des dynamiques Y82-MD1 et T82-MD2 montrent que le TEA reste lié à la bouche externe du canal sauvage mais s'échappe rapidement (après $1.1 \mathrm{~ns}$ ) du canal muté. De plus, même pendant la première nanoseconde, le TEA parait lié de manière plus forte au canal sauvage qu'au canal muté: la position moyenne du TEA est de $Z=21.4 \AA$ pendant la trajectoire Y82-MD1 et de $Z=24.0 \AA$ pendant la simulation T82-MD1; le TEA se lie donc $3 \AA$ plus profondément à l'intérieur du canal présentant un résidu aromatique en position 82 . La conformation globale du canal n'est pas affectée par une simple mutation en position 82. La différence RMS entre les coordonnées du squelette des canaux sauvage et mutant, moyennées sur 500 ps, entre les résidus 77 et 84 est de l'ordre de $1.4 \AA$ A. C'est un peu plus que les différences observées entre les divers monomères d'un même canal $(0.3$ à $0.9 \AA)$ Bien que restant associé à l'entrée extracellulaire du canal KcsA-Y82, le TEA subit de rapides mouvements de rotation. De plus, une transition spontanée et concertée des ions $\mathrm{K}^{+}$dans le site interne et la cavité est enregistrée pendant les premières 100 ps de la période de production dans les 2 simulations Y82MD1 et T82-MD1 Lors de la transition, l'ion $\mathrm{K}^{+}$du site interne s'est déplacé vers le site externe

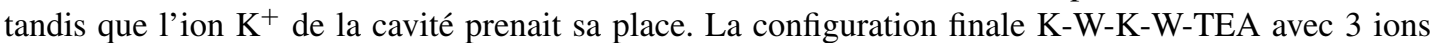
dans le filtre de sélectivité rappelle les résultats déjà observés dans des simulations de DM du canal KcsA [3].

Les résultats précédents sont en accord avec les observations expérimentales [13]: le blocage côté extracellulaire par le TEA est plus stable en présence de résidu aromatique en position équivalente 449 chez le Shaker. Le calcul du PMF pour l'association du TEA avec l'entrée extracellulaire du canal va 
permettre de caractériser le blocage de manière quantitative. En particulier, les constantes de liaison peuvent s'exprimer en fonction du PMF [24].

La Fig. 5 (à gauche) montre les PMF calculés pour la liaison du TEA coté extracellulaire des canaux KcsA-Y82 et KcsA-T82, $\mathcal{W}_{\mathrm{Y} 82}(r)$ et $\mathcal{W}_{\mathrm{T} 82}(r)$.

Pour le canal KcsA-Y82, il y a un premier puits d'énergie libre en 9.85 Å suivi d'un second autour de $12 \AA$ Å. Pour KcsA-T82, il y a un puits unique situé en $11.55 \AA$ ( $r$ correspond à la distance entre le centre de masse du TEA et le centre de masse des résidus formant le filtre de sélectivité). L'état lié du TEA est clairement plus stable dans le cas KcsA-Y82. L'énergie libre de liaison relative $\Delta G \equiv G_{\mathrm{T} 82}-G_{\mathrm{Y} 82}$ est donnée par

$$
\Delta G=-k_{B} T \ln \left[\frac{\int_{0}^{r_{\mathrm{c}}} d r \exp \left[-\mathcal{W}_{\mathrm{T} 82}(r) / k_{\mathrm{B}} T\right]}{\int_{0}^{r_{\mathrm{c}}} d r \exp \left[-\mathcal{W}_{\mathrm{Y} 82}(r) / k_{\mathrm{B}} T\right]}\right]
$$

où $r_{\mathrm{c}}$, fixé à $14 \AA$ A , est la distance de coupure qui permet de définir l'état lié.

Par l'équation Eq. (4.1), on arrive à une différence d'énergie libre de $+2.1 \mathrm{kcal} / \mathrm{mol}$, à comparer à la valeur expérimentale équivalente mesurée sur le canal Shaker: $\mathrm{kT} \log (22 / 0.65)=+2.1 \mathrm{kcal} / \mathrm{mol}$ [13].

Deux nouvelles simulations de DM de 0.5ns (Y82-MD2 et T82-MD2) ont été réalisées avec le TEA situé initialement dans les puits d'énergie libre calculés précédemment. On observe dans le cas du canal KcsA-Y82 que le TEA se comporte comme un véritable bouchon avec un plan principal quasiperpendiculaire à l'axe du canal et des carbones terminaux orientés vers les tyrosines. Le TEA passe un temps à peu près équivalent en contact avec les tyrosines de chacun des 4 monomères en accord avec les résultats expérimentaux [13]. Il apparait au contraire penché et décentré dans le canal KcsA-T82.

Dans le canal KcsA-Y82, le TEA ne semble pas interagir directement avec le plan des chaines latérales aromatiques: les carbones terminaux du TEA sont proches des carbones CE2 des cycles des tyrosines avec une distance moyenne de $4.5 \AA$. L'énergie des interactions de van der Waals directes entre TEA et chaines latérales des tyrosines est de l'ordre de $-0.6 \mathrm{kcal} / \mathrm{mol}$. Ces observations suggèrent que l'affinité accrue du TEA pour le canal présentant un cycle aromatique n'est pas due directement à des interactions П-cation [25] comme cela a pu être proposé [13].

Comme le TEA est un gros cation "hydrophobe", les forces d'hydratation peuvent jouer un rôle important dans la stabilization du blocage. Nous avons donc calculé la distribution radiale des oxygènes des molécules d' eau autour de l'atome d'azote du TEA. Les résultats sont visibles sur la Fig. 5 (à droite) en comparaison avec la fonction de distribution calculée pour le TEA dans l'eau pure. Clairement, la densité de molécules d'eau autour du TEA est maximale dans l'eau pure, minimale dans la simulation KcsAY82 et intermédiaire dans la simulation KcsA-T82. Il y a un nombre sensiblement égal de molécules
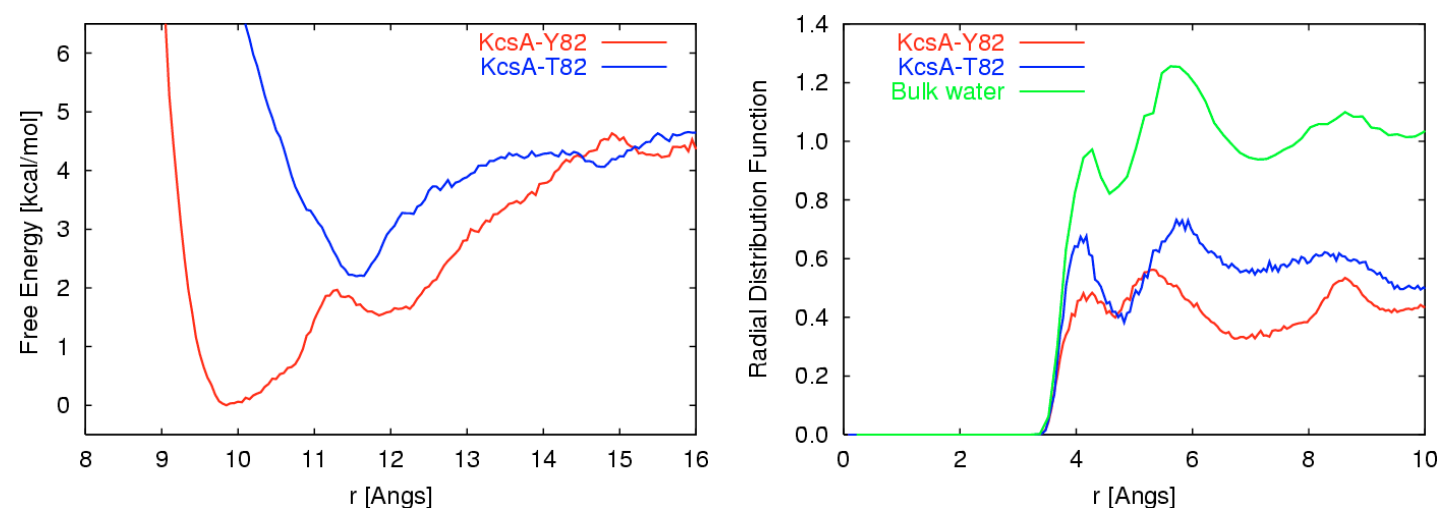

Figure 5. A gauche: Potentiels de force moyenne pour la liaison du TEA côté extracellulaire des canaux KcsA-Y82 et KcsA-T82; à droite: Distributions radiales des oxygènes des molécules d'eau autour de l'atome d'azote du TEA. 
d'eau autour du TEA dans la première sphère d'hydratation $(0<r<4.8 \AA$ A $)$ pour les canaux KcsAY82 et KcsA-T82, respectivement. Par contre, dans la seconde sphère $(4.8<r<7.3 \AA)$, il y a 16 molécules d'eau autour du TEA dans la simulation KcsA-Y82, contre 28 dans la simulation KcsAT82. (On en compte 8.5 et 41 dans la première et la deuxième couche respectivement, dans l'eau pure). Il y a donc environ 7 molécules d'eau en plus autour du TEA dans le canal mutant que dans le canal sauvage principalement à cause d'un meilleur ajustement de l'ion à l'entrée du canal sauvage qui laisse peu de place à des molécules d'eau intercalées. Ceci suggère que la structure d'hydratation du TEA pourrait être responsable de la différence de stabilité du blocage extracellulaire révélé par les calculs de PMF.

\section{CONCLUSION}

Nous avons donc montré briévement comment les simulations de Dynamique Moléculaire pouvaient être utilisées afin de comprendre les mécanismes responsables de l'ouverture ou de la fermeture des canaux ioniques et de manière générale certains processus bi-moléculaires dans les protéines. Il ne faut jamais perdre de vue que cette analyse mécanistique repose sur une validation préalable du modèle par une confrontation avec des données expérimentales. La diffusion des neutrons est une technique expérimentale particulièrement bien adaptée à cette fin: elle peut donner accès à la position des protons, à des rayons de giration, à des fonctions de corrélation ou fonctions de distribution de paires etc., des résultats qui peuvent être utilisés directement, comparés ou recalculés à partir des simulations. Les mesures d'affinité tirées d'expériences de biochimie ou d'électrophysiologie peuvent également, comme nous l'avons vu, mais au prix d'efforts de calcul importants servir de base à cette validation.

Merci à Benoit Roux, (Université Cornell, New York) pour d'intéressantes discussions, aux organisateurs des JDN XII pour leur aimable invitation et au CEA pour l'accès aux moyens de calcul centralisés.

\section{Références}

[1] S. Crouzy, T. B. Woolf and B. Roux. A molecular dynamics study of gating in dioxolane-linked gramicidin A channels. Biophys. J., 67:1370-1386, 1994.

[2] S. Crouzy, S. Bernèche and B. Roux. Extracellular blockade of $\mathrm{K}^{+}$channels by TEA: results from molecular dynamics simulations of the KcsA channel. J. Gen. Physiol., 118:207-217, 2001.

[3] S. Berneche and B. Roux. Molecular dynamics of the KcsA K(+) channel in a bilayer membrane. Biophys J, 78:2900-2917, 2000.

[4] B. Roux and R. MacKinnon. The cavity and pore helices in the $\mathrm{K} c s \mathrm{~A} \mathrm{~K}^{+}$channel: electrostatic stabilization of monovalent cations. Science, 285:100-102, 1999.

[5] G. M. Torrie and J. P. Valleau. Monte Carlo free energy estimates using non-Boltzmann sampling: application to the sub-critical Lennard-Jones fluid. Chem. Phys. Let., 28, october 1974.

[6] B. Roux. The calculation of the potential of mean force using computer simulations. Comp. Phys. Comm., 91:275-282, 1995.

[7] M. Ortega, M. K. Sautriau, J. D. Lafontaine and S. Crouzy. MPV3D: a program for the display of Macromolecules and Proteins in 3 Dimensions. 2004.

[8] C. J. Stankovic, S. H. Heinemann, J. M. Delfino, F. J. Sigworth and S. L. Schreiber. Tranmembrane Channels Based on Tartaric Acid-Gramicidin A Hybrids. Science, 244:813-817, 1989.

[9] A. S. Arseniev, I. L. Barsukov and V. F. Bystrov. N. M. R. solution structure of gramicidin A complex with caesium cations. FEBS Lett., 180:33-39, 1985.

[10] S. Crouzy, J. Baudry, J. C. Smith and B. Roux. Efficient calculation of two-dimensional adiabatic and free energy maps: Application to the isomerization of the $\mathrm{C} 13=\mathrm{C} 14$ and $\mathrm{C} 15=\mathrm{N} 16$ bonds in the retinal of bacteriorhodopsin. J. Comp. Chem., 20:1644-1658, 1999. 
[11] S. H. Heinemann and F. J. Sigworth. Open channel Noise V. A fluctuating barrier to ion entry in Gramicidin A channels. Biophys. J., 57:499-514, 1990.

[12] C. M. Armstrong. Inactivation of the potassium conductance and related phenomena caused by quaternary ammonium ion injection in squid axons. J Gen Physiol, 54:553-575, 1969.

[13] L. Heginboham and R. MacKinnon. The aromatic binding site for tetraethylammonium ion on potassium channels. Neuron, 8:483-491, 1992.

[14] D. A. Doyle, J. M. Cabral, R. A. Pfuetzner, A. Kuo, J. M. Gulbis, S. L. Cohen, B. T. Chait and R. MacKinnon. The structure of the potassium channel: molecular basis of $\mathrm{K}+$ conduction and selectivity. Science, 280:69-77, 1998.

[15] D. Meuser, H. Splitt, R. Wagner and H. Schrempf. Exploring the open pore of the potassium channel from Streptomyces lividans. FEBS Lett, 462:447-452, 1999.

[16] R. MacKinnon, S. L. Cohen, A. Kuo, A. Lee and B. T. Chait. Structural conservation in prokaryotic and eukariotic potassium channels. Science, 280:106-109, 1998.

[17] B. R. Brooks, R. E. Bruccoleri, B. D. Olafson, D. J. States, S. Swaminathan and M. Karplus. CHARMM: a program for macromolecular energy, minimization, and dynamics calculations. $J$. Comp. Chem., 4:187-217, 1983.

[18] A. D. Jr. MacKerell, D. Bashford, M. Bellot, R. L. Dunbrack, J. D. Evanseck, M. J. Field, S. Fischer, J. Gao, H. Guo, D. Joseph-McCarthy, S. Ha, L. Kuchnir, K. Kuczera, F. T. K. Lau, C. Mattos, S. Michnick, T. Ngo, D. T. Nguyen, B. Prodhom, W. E. Reiher III, B. Roux, M. Schlenkrich, J. Smith, R. Stote, J. Straub, M. Watanabe, J. Wiorkiewicz-Kuczera and M. Karplus. All-atom empirical potential for molecular modeling and dynamics studies of proteins. J. Phys. Chems. B, 102:3586-3616, 1998.

[19] M. J. Schlenkrich, J. Brickmann, A. D. Jr. MacKerell and M. Karplus. An empirical potential energy function for phospholipids: Criteria for parameters optimization and applications. In K.M. Merz and B. Roux, editors, Biological Membranes. A molecular perspective from computation and experiment, pages 31-81. Birkhauser, Boston, 1996.

[20] D. Beglov and B. Roux. Finite representation of an infinite bulk system: Solvent Boundary Potential for Computer Simulations. J. Chem. Phys., 100:9050-9063, 1994.

[21] D. H. Aue, H. M. Webb and M. T. Bowers. A thermodynamic analysis of solvation effects on the basicity of alkylamine. An electrostatic analysis of subtituents effects. JAm Chem Soc, 98:318-329, 1976.

[22] S. E. Feller, Y. H. Zhang, R. W. Pastor and B. R. Brooks. Constant pressure molecular dynamics simulation - the Langevin piston method. Journal of Chemical Physics, 103:4613-4621, 1995.

[23] U. Essmann, L. Perera, M. L. Berkowitz, T. Darden, H. Lee and L. G. Pedersen. A smooth particle mesh Ewald method. Journal of Chemical Physics, 103:8577-8593, 1995.

[24] M. K. Gilson, J. A. Given, B. L. Bush and J. A. McCammon. The statistical-thermodynamic basis for computation of binding affinities: A critical review. Biophys. J., 72:1047-1069, 1997.

[25] J. Sunner, K. Nishizawa and P. Kebarle. Ion-solvent molecule interactions in the gas phase. The potassium ion and benzene. J Phys. Chem., 85:1814-1820, 1981. 